\title{
Generalized Abel Inversion Using Homotopy Perturbation Method
}

\author{
Sunil Kumar, Om P. Singh, Sandeep Dixit \\ Department of Applied Mathematics, Institute of Technology, Banaras Hindu University, Varanasi, India \\ E-mail: skiitbhu28@gmail.com,singhom@gmail.com, sandydixit27@gmail.com \\ Received October 19, 2010; revised December 28, 2010; accepted January 3, 2011
}

\begin{abstract}
Many problems in physics like reconstruction of the radially distributed emissivity from the line-of-sight projected intensity, the 3-D image reconstruction from cone beam projections in computerized tomography, etc. lead naturally, in the case of radial symmetry, to the study of Abel's type integral equation. Obtaining the physically relevant quantity from the measured one requires, therefore the inversion of the Abel's integral equation. The aim of this letter is to present a user friendly algorithm to invert generalized Abel integral equation by using homotopy perturbation method. The stability of the algorithm is analysed. The validity and applicability of this powerful technique is illustrated through various particular cases which demonstrate its efficiency and simplicity in solving these types of integral equations.
\end{abstract}

Keywords: Generalized Abel Integral Equation, Homotopy Perturbation Method, Noise Term, Stability

\section{Introduction}

Since Abel formulated his integral equation [1] and presented its analytic solution, the equation has found application in many branches of physical science. The earliest application, due to Mach [2], arose in the study of compressible flows around axially symmetric bodies. Usually, physical quantities accessible to measurement are quite often related to physically important but experimentally inaccessible ones by Abel's integral equation, [3-9]. Obtaining the physically relevant quantity from the measured one requires, therefore, the inversion of the Abel's integral equation, and in case the object does not have radial symmetry, it requires, in principal, the inversion of Random transform.

We consider the following generalized Abel's integral equation

$$
\int_{0}^{x} \frac{y(t)}{(x-t)^{\alpha}} d t=g(x), 0<\alpha<1, x>0,
$$

where $g(x)$ is the known function. The expression $(x-t)^{-\alpha}$ is called the kernel of the Abel's integral equation or Abel kernel. An Abel's integral equation belongs to the class of Volterra equation of the first kind. If $g(x)$ is a continuously differentiable function, then the Abel's integral Equation (1) has a unique solution

$$
y(x)=\frac{\sin (\alpha \pi)}{\pi} \frac{d}{d x} \int_{0}^{x} \frac{g(t)}{(x-t)^{1-\alpha}} d t, \quad[10]
$$

which is equivalent to

$$
y(x)=\frac{\sin (\alpha \pi)}{\pi}\left[\frac{g(0)}{x^{1-\alpha}}+\int_{0}^{x} \frac{g^{\prime}(t)}{(x-t)^{1-\alpha}} d t\right] .
$$

Though, while the analytic solution to the Abel Equation (1) is given by (2), in practice we have only a point wise approximation to g, so the inversion must be carried out numerically. Since the integral transform (2) is equivalent to fractional differentiation of order $1-\alpha$ some amplification of data noise is inevitable [11]. As the process of estimating the solution function $y(x)$, if the data function $g(x)$ is given approximately and only at a discrete set of data points, is ill-posed since even very small, high frequency errors in the measured $g(x)$, such as will arise from experimental errors, photon counting noise and noise in the electronics, might cause large errors in the reconstructed solution $y(x)$. This is due to the fact that inversion formula (3) requires differentiating the measured data $g(x)$. In 1982, an analytic but derivative free inversion formula was obtained by Deutsch and Beniaminy [12] to avoid this problem. In addition, many numerical inversion methods [13-21] have been developed with varying degree of success with the inherent limita- 
tions of all measured data. Consequently, the direct use of (2) and (3) are restricted and stable numerical methods become important.

The aim of the present letter is to propose an algorithm to invert the Abel's integral Equation (1) by using the homotopy perturbation method (HPM). We construct a convex homotopy by using HPM to obtain an iterative solution to (1) and analyze the stability of the algorithm. Some numerical examples are also presented to illustrate the accuracy of the algorithm.

\section{Method of Solution}

In this method, using the homotopy technique of topology, a homotopy is constructed with an embedding parameter $p \in[0,1]$, which is considered as a "small parameter". When the homotopy theory is coupled with perturbation theory it provides a powerful mathematical tool. The details of the method can be found in [22-24].

We construct the following convex homotopy

$$
(1-p) L(x)+p\left[\int_{0}^{x} \frac{L(t)}{(x-t)^{\alpha}} d t-g(x)\right]=0,
$$

to develop a numerical inversion algorithm for the Abel integral Equation (1), where the embedding parameter $p \in[0,1]$ can be consider as an expanding parameter [23], to obtain

$$
L(x)=\sum_{i=0}^{\infty} p^{i} L_{i}(x),
$$

where, $L_{i}(x), i=0,1,2,3, \cdots$ are the functions to be determined. We use the following iterative scheme to evaluate $L_{i}(x)$.

Substituting (5) in (4) and the equating the coefficients of $p$ with the same power, we get

$$
\begin{aligned}
& p^{0}: L_{0}(x)=0, p^{1}: L_{1}(x)=g(x), \\
& p^{2}: L_{2}(x)=L_{1}(x)-\int_{0}^{x} \frac{L_{1}(t)}{(x-t)^{\alpha}} d t, \\
& p^{3}: L_{3}(x)=L_{2}(x)-\int_{0}^{x} \frac{L_{2}(t)}{(x-t)^{\alpha}} d t, \cdots \\
& p^{n}: L_{n}(x)=L_{n-1}(x)-\int_{0}^{x} \frac{L_{n-1}(t)}{(x-t)^{\alpha}} d t .
\end{aligned}
$$

Hence the solution of Equation (1) is given by

$$
y(x)=\lim _{p \rightarrow 1} L(x)=\sum_{i=0}^{\infty} L_{i}(x) .
$$

Now, we consider the stability of the solution (7) to small changes in data. That is we are interested in what happens to $y$, when we replace $g(x)$ by $g(x)+\delta g(x)$, where $\delta g(x)$, is unknown apart from some restriction on its magnitude relative to $g(x)$. For computational convenience, we write $\delta g(x)=\varepsilon_{1}(x)$. Subsequently, the iterative scheme (6) becomes

$$
\begin{aligned}
& \tilde{L}_{0}(x)=0, \\
& \tilde{L}_{1}(x)=g(x)+\varepsilon_{1}(x), \\
& \tilde{L}_{2}(x)=L_{2}(x)+\varepsilon_{1}(x), \cdots \\
& \tilde{L}_{n}(x)=L_{n}(x)+\varepsilon_{n}(x),
\end{aligned}
$$

where $L_{n}(x)$ is given by (6) and $\varepsilon_{n}(x)=\varepsilon_{n-1}(x)-\int_{0}^{x} \frac{\varepsilon_{n-1}(t)}{(x-t)^{\alpha}} d t$, for $\mathrm{n}=2,3,4, \cdots$

Thus, the new solution $\tilde{y}(x)$, is given by

$$
\tilde{y}(x)=\lim _{n \rightarrow \infty} \sum_{i=0}^{n} \tilde{L}_{i}(x) .
$$

The effect of the noise $\varepsilon_{1}(x)$ in the data deviates the solution by

$$
\begin{aligned}
\delta y(x) & =\tilde{y}(x)-y(x)=\lim _{n \rightarrow \infty} \sum_{i=0}^{n}\left[\tilde{L}_{i}(x)-L_{i}(x)\right] \\
& =\lim _{n \rightarrow \infty} \sum_{i=0}^{n} \varepsilon_{i}(x),
\end{aligned}
$$

where $\varepsilon_{1}(x)=0$.

From (10), we conclude that $\delta y$ and $\delta g$ are connected via the following generalized Abel integral equation

$$
\int_{0}^{x} \frac{\delta y(t)}{(x-t)^{\alpha}} d t=\delta g(x) .
$$

Thus, we have proved the following theorem

Theorem. The presence of a noise term $\delta g(x)$ in the observable data $g(x)$ changes the solution $y(x)$ by an amount equivalent to the solution of the Abel integral Equation (11) with input equal to the noise term $\delta g(x)$ itself.

As $\delta g(x)$ is not known before hand, we take an upper bound for $\delta g(x)$. Let $\sup _{0<x<1}|g(x)|=\varepsilon$, then (11) reduces to $\int_{0}^{x} \frac{\delta y(t)}{(x-t)^{\alpha}} d t=\varepsilon$, which can be readily solved.

\section{Numerical Examples}

The simplicity and accuracy of the proposed algorithm is illustrated by the following numerical examples. We compute the error $E(x)=[y(x)-\hat{y}(x)]$, where $y(x)$ is the exact solution and $y(x)$ is an approximate solution of the problem obtained by truncating equation (7).

Examples. To illustrate the method, we consider the 
following three pairs of generalized Abel integral equations with their inversions:

$$
\int_{0}^{x} \frac{y(t)}{(x-t)^{2 / 3}} d t=9 x_{2}^{4 / 3} F_{2}\left[\left\{\frac{1}{2}, 1\right\},\left\{\frac{7}{6}, \frac{5}{3}\right\},-x^{2}\right],
$$

with the exact solution $y(x)=2 \sqrt{\pi} \operatorname{erf}(x)$,

$$
\int_{0}^{x} \frac{y(t)}{(x-t)^{1 / 2}} d t=x e^{-x / 2}\left[I_{0}\left(\frac{x}{2}\right)+I_{1}\left(\frac{x}{2}\right)\right],
$$

with exact solution $y(x)=\frac{\operatorname{erf}(\sqrt{x})}{\sqrt{\pi}}$, where $I_{n}(x)$

and $\operatorname{erf}(x)$ are the modified Bessel function of first kind and the error function respectively.

$$
\begin{aligned}
& \int_{0}^{x} \frac{y(t)}{(x-t)^{1 / 3}} d t=\frac{15 \sqrt{\pi} \Gamma\left(\frac{2}{3}\right)}{8 \Gamma\left(\frac{25}{6}\right)} x^{19 / 6}, \\
& \text { with exact solution } y(x)=x^{5 / 2} .
\end{aligned}
$$

Using the iterative scheme (6) and truncating the solution series (7) at levels $n=13,27$ and 37 for the pairs (14), (15) and (16) respectively; we obtain the approximate solutions of the above problems. The various errors $E_{i}(x), i=1,2,3$ are shown in the Figure 1.

\section{Conclusions}

A very simple but powerful and user friendly algorithm to invert generalized Abel integral equation is proposed by using homotopy perturbation method. It is proved that the change $\delta y(x)$, in the solution $y(x)$ caused by the presence of noise $\delta g(x)$ in the observable data $g(x)$, is the solution of the generalized Abel integral equation with input data equal to the noise $\delta g(x)$ itself.

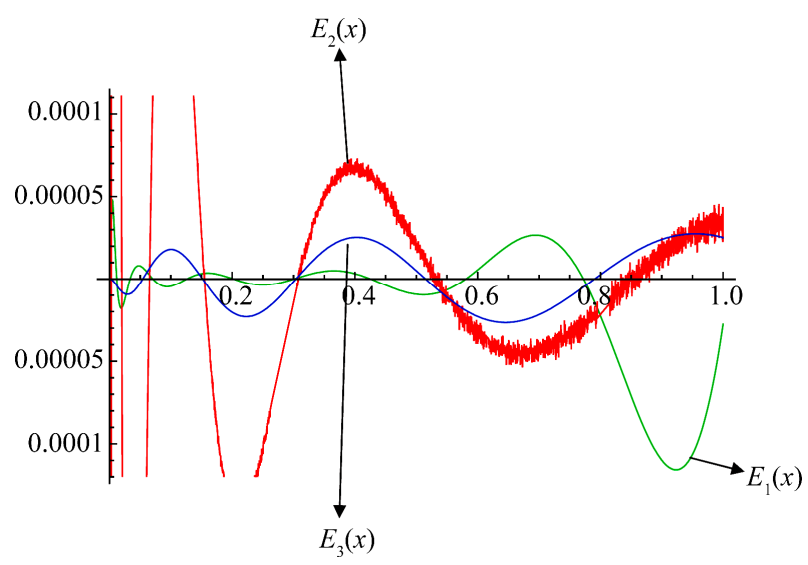

Figure 1. The various errors $E_{i}(x), i=1,2,3$ for the pairs of Abel's integral Equations (14)-(16).

\section{Acknowledgements}

The first author acknowledges the financial supports from Rajiv Gandhi National Fellowship of the University Grant Commission, New Delhi.

\section{References}

[1] N. H. Abel, "Resolution d'un Probleme,” Journal fur die reine und angewandte Mathematik, Vol. 1, 1826, pp. 153157. doi:10.1515/crll.1826.1.153

[2] L. Mach, Wiener Akademie Berlin fur Mathematik und Physik Klasse, Vol. 105, 1896, p. 605.

[3] S. B. Healy, J. Haase and O. Lense, "Abel Transform Inversion of Radio Occultation Measurement Made with a Receiver inside the Earth's Atmosphere," Annales Geophysicae, Vol. 20, 2002, p. 1253. doi:10.5194/angeo-20-1253-2002

[4] R. N. Bracewell and A. C. Riddle, "Inversion of FanBeam Scans in Radio Astronomy,” Astrophysical Journal, Vol. 150, 1967, pp. 427- 434. doi:10.1086/149346

[5] S. C. Soloman, P. B. Hays and V. J. Abreu, "Tomographic Inversion of Satellite Photometry,” Applied Optics, Vol. 23, 1984, pp. 3409-3414. doi:10.1364/AO.23.003409

[6] E. L. Kosarev, "Applications of Integral Equations of the First Kind in Experiment Physics,” Computer Physics Communication, Vol. 20, 1980, pp. 69-75. doi:10.1016/0010-4655(80)90110-1

[7] C. J. Tallents, M. D. J. Burgess and B. Luther-Davies, "The Determination of Electron Density Profile from Refraction Measurements Obtained Using Holographic Interferometry," Optics Communications, Vol. 4, 1983, pp. 384-387. doi:10.1016/0030-4018(83)90222-5

[8] L. J. M. Ignjatovic and A. A. Mihajlov, "The Realization of Abel's Inversion in the Case of Discharge with Undetermined Radius," Journal of Quantitative Spectroscopy \& Radiative Transfer, Vol. 72, 2002, pp. 677-689. doi:10.1016/S0022-4073(01)00149-2

[9] M. J. Buie, J. T. P. Pender, J. P. Holloway, T. Vincent, P. L. G. Ventzek and M. L. Brake, “Abel Inversion Applied to Experimental Spectroscopic Data with Off Axis Peaks," Journal of Quantitative Spectroscopy \& Radiative Transfer, Vol. 55, No. 2, 1996, pp. 231-243. doi:10.1016/0022-4073(95)00149-2

[10] F. G. Tricomi, "Integral Equations”, Interscience, New York, 1975.

[11] G. N. Minerbo and M. E. Levy, "Inversion of Abel Integral Equation by Means of Orthogonal Polynomials," SIAM Journal of Computational Physics, Vol. 6, 1969, pp. 598-616.

[12] M. Deutsch and I. Beniaminy, "Derivatives Free Inversion of Abel's Integral Equations,” Applied Physics Letters, Vol. 41, 1982, pp. 27-28. doi:10.1063/1.93309

[13] M. Deutsch, A. Notea and D. Pal, "Inversion of Abel's Integral Equations and Its Application to NDT by X-Ray 
Radiography,” NDT International, Vol. 23, No. 1, 1990, pp. 32-38.

[14] J. P. Lanquart, "Error Attenuation in Abel Inversion," Journal of Computational Physics, Vol. 47, 1982, pp. 434-443. doi:10.1016/0021-9991(82)90092-4

[15] L. J. M. Ignjatovic and A. A. Mihajlov, "The Realization of Abel's Inversion in the Case of Discharge with Undetermined Radious," Journal of Quantitative Spectroscopy \& Radiative Transfer, Vol. 72, 2002, pp. 677-689. doi:10.1016/S0022-4073(01)00149-2

[16] V. K. Singh, R. K. Pandey and O. P. Singh, "New Stable Numerical Solution of Singular Integral Equations of Abel Type by Using Normalized Bernstein Polynomials,” Applied Mathematical Sciences, Vol. 3, No. 5, 2009, pp. 241-255.

[17] O. P. Singh, V. K. Singh and R. K. Pandey, "New Stable Numerical Inversion of Abel Integral Equation Using Almost Bernstein Operational Matrix," Journal of Quantitative Spectroscopy \& Radiative Transfer, Vol. 111, 2010, pp. 245-252. doi:10.1016/j.jqsrt.2009.07.007

[18] I. Beniaminy and M. Deutsch, "Abel Stable High Accuracy Programme for the Inversion of Abel's Integral Equation,” Computer Physics Communication, Vol. 27, 1982, pp. 415-422. doi:10.1016/0010-4655(82)90102-3

[19] D. A. Murio, D. G. Hinestroza and C. E. Mejia, "New
Stable Numerical Inversion of Abel's Integral Equation," Computers and Mathematics with Applications, Vol. 23, No. 11, 1992, pp. 3-11. doi:10.1016/0898-1221(92)90064-O

[20] S. Ma, H. Gao, L. Wu and G. Zhang, "Abel Inversion Using Legendre Polynomials Approximations,” Journal of Quantitative Spectroscopy \& Radiative Transfer, Vol. 109, 2008, pp. 1745-1757. doi:10.1016/j.jqsrt.2008.01.013

[21] S. Ma, H. Gao, G. Zhang and L. Wu, "Abel Inversion Using Legendre Wavelet Expansion,” Journal of Quantitative Spectroscopy \& Radiative Transfer, Vol. 107, 2007, pp. 61-71. doi:10.1016/j.jqsrt.2007.01.054

[22] J. H. He, “An Approximate Solution Technique Depending upon an Artificial Parameter," Communications in Nonlinear Science and Numerical Simulation, Vol. 3, No. 2, 1998, pp. 92-97. doi:10.1016/S1007-5704(98)90070-3

[23] J. H. He, “Homotopy Perturbation Technique,” Computer Methods in Applied Mechanics and Engineering, Vol. 178, 1999, pp. 257-262. doi:10.1016/S0045-7825(99)00018-3

[24] J. H. He, "A Review on Some Recently Developed Nonlinear Analytical Technique,” International Journal of Nonlinear Sciences and Numerical Simulation, Vol. 1, No. 1, 2000, pp. 51-70. 